\title{
Expression of miR-30c and miR-29b in prostate cancer and its diagnostic significance
}

\author{
CHUANAN ZHU ${ }^{1}$, XIUMEI HOU ${ }^{2}$, JIABIN ZHU ${ }^{3}$, CHUNXIAO JIANG ${ }^{1}$ and WEI WEI ${ }^{1}$ \\ ${ }^{1}$ Department of Clinical Laboratory, Jining First People's Hospital, Jining, Shandong 272000; \\ ${ }^{2}$ Nursing Department, Jining Psychiatric Hospital, Jining, Shandong 272051; ${ }^{3}$ Department of Neurology, \\ The General Hospital of Ningxia Medical University, Yinchuan, Ningxia 750000, P.R. China
}

Received February 12, 2018; Accepted June 14, 2018

DOI: $10.3892 / \mathrm{ol} .2018 .9007$

\begin{abstract}
This study aimed to investigate the expression of miR-30c and miR-29b in prostate cancer (PCa) and its clinical significance. The expression of miR-30c and miR-29b was detected by RT-qPCR in 187 cases of PCa and their adjacent tissues. Combined with clinical information, the correlation between the expression of miR-29b and miR-30c and the clinical features of PCa was analyzed, and ROC curve was plotted. The expression of miR-30c and miR-29b detected by RT-qPCR showed that the expression of miR-29b and miR-30c in PCa tissues was significantly lower than that in adjacent cancerous tissues $(\mathrm{p}<0.05)$. By comparing the expression and clinical data of miR-29b and miR-30c in the cancer tissues of PCa patients, it was observed that age, smoking, and TNM staging were not related to miR-29b and miR-30c expression ( $p>0.05)$, while lymph node metastasis, bone metastasis, and Gleason score were related to the expression of miR-29b and miR-30c $(\mathrm{p}<0.01)$. The ROC curve showed that miR-29b AUC, 0.924; 95\% CI, 0.824-0.967, and miR-30c AUC, 0.944; 95\% CI, 0.798-0.972. miR-30c and miR-29b are clinically relevant to $\mathrm{PCa}$. In conclusion, detecting the expression of miR-30c and miR-29b not only can differentiate between PCa and paracancerous tissues, but it is also anticipated to become a new biomarker for the diagnosis of PCa.
\end{abstract}

\section{Introduction}

Prostate cancer ( $\mathrm{PCa}$ ), one of the malignant tumors of the prostate gland, is frequently found in the lateral and posterior lobes of the prostate as well as in the glands. It has a high incidence in the middle-aged and elderly men. It ranks 3rd among the highest incidence of male tumors. The mortality rate ranks 6th, and there are also regional differences in incidence of PCa.

Correspondence to: Dr Wei Wei, Department of Clinical Laboratory, Jining First People's Hospital, 6 Jiankang Road, Rencheng, Jining, Shandong 272000, P.R. China

E-mail:w2uqjd@163.com

Key words: miR-30c, miR-29b, prostate cancer, miRNA
According to statistics, the global incidence and mortality gap is approximately 25 times and 10 times, respectively (1-3). Another study shows that (4), it is speculated that by 2030 in China PCa incidence and mortality will double on the existing basis, an average of $>5 \%$ annual increase. At present, the early diagnosis of $\mathrm{PCa}$ is mainly biopsy and serum prostate specific antigen (PSA), but in the paracancerous benign prostatic hyperplasia and prostatitis, the PSA expression will also increase, indicating that PSA has a high sensitivity but poor specificity, which may lead to some bias towards the diagnosis of clinicians, and the presence of gray areas makes it not a good indicator of the prognosis of patients $(5,6)$. Thus, we need to find a way to diagnose PCa, to reduce the clinical misdiagnosis and missed diagnosis.

MicroRNAs (miRNAs) are a class of endogenous non-coding single-stranded small molecule RNAs that regulate the expression of target genes at the post-transcriptional level, and are involved in the regulation of various physiological and pathological functions (7). Bioinformatics data show that a single miRNA can bind to hundreds of target mRNAs, and thus play an important role in various biological processes (8). There is growing evidence that there is a link between miRNAs and tumorigenesis. miR-29b and miR-30c are members of the miRNA family. TCGA database indicated that miR-29b and miR-30c show low expression in PCa cancer tissues.

Therefore, we explored the expression of miR-29b and miR-30c in PCa tissue, in order to provide a basis for the diagnosis and treatment of PCa.

\section{Materials and methods}

For this investigation cancer tissues and adjacent tissues of 187 cases of PCa by prostatectomy were collected from March 2011 to February 2013 in the Department of Urology of Jining First People's Hospital (Jining, China). Samples were excised and frozen in liquid nitrogen within $5 \mathrm{~min}$ for preservation. There was a total of 187 male patients, aged from 47-65 years, mean age 52.05 \pm 8.47 years. Patients were divided according to the 7th edition of TNM staging standard of 2009; PCa by Union for International Cancer Control (UICC) (9): 55 cases in stage I, 60 cases in stage II and 72 cases in stage III-IV. Pathological type of all specimens obtained was PCa. Clinical specimens were collected with the approval 
Table I. Primer sequences.

\begin{tabular}{lll}
\hline Genes & \multicolumn{1}{c}{ Upstream sequences } & \multicolumn{1}{c}{ Downstream sequences } \\
\hline U6 internal reference & 5'-CTCGCTTCGGCAGCACA-3' & 5'-AACGCTTCACGAATTTGCGT-3' \\
miR-29b & 5'-GCGCGCTAGCACCATTTG-3' & 5'-CAGTGCAGGGTCCGAGGT-3' \\
miR-30c & 5'--3' & 5'-AGATGAGCATTGGCAGCGAG-3'
\end{tabular}

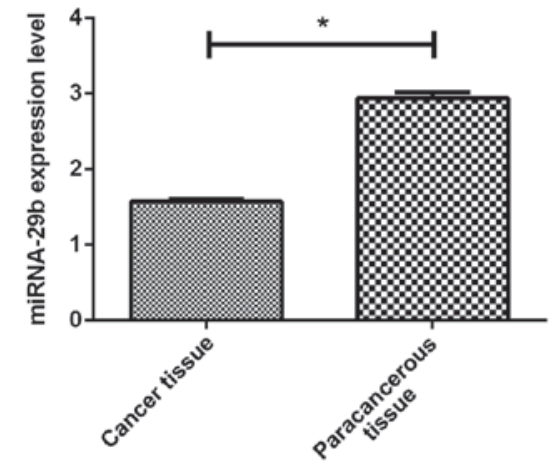

Figure 1. Expression of miRNA-29b in PCa tissues. miRNA-29b was found to be significantly lower in cancerous tissues of prostate patients $(1.57 \pm 0.48$ vs. $2.94 \pm 1.08)$ by RT-qPCR $\left(\mathrm{t}=5.85,{ }^{*} \mathrm{P}<0.001\right) . \mathrm{PCa}$, prostate cancer.

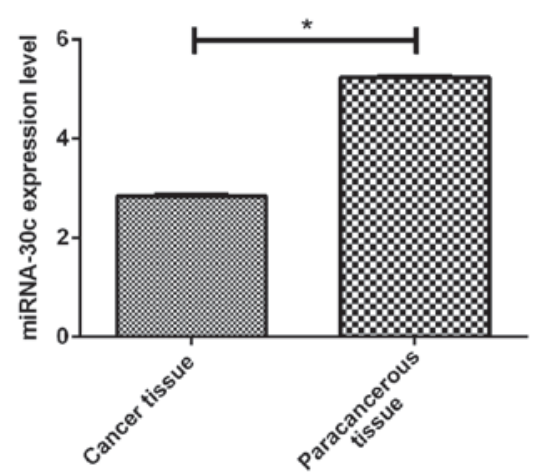

Figure 2. Expression of miRNA-30c in PCa tissue RT-qPCR miRNA-30c is significantly lower in prostate cancer tissues $(2.84 \pm 0.57)$ than that in paracancerous tisues $\left(5.24 \pm 0.42, \mathrm{t}=46.45,{ }^{*} \mathrm{P}<0.001\right)$. $\mathrm{PCa}$, prostate cancer.

of Jining First People's Hospital Medical Ethics Committee (Jining, China), and patients signed informed consent.

The main reagents. TRIzol reagent and miRNA reverse transcriptase kit were purchased from Invitrogen company (Thermo Fisher Scientific, Inc., Carlsbad, CA, USA); SYBR-Green Master Mix was purchased from Applied Biosystems (Thermo Fisher Scientific, Inc.). ABI StepOne Plus fluorescence quantitative PCR instrument was purchased from Applied Biosystems. NanoDrop 2000 spectrophotometer, miR-29b and miR-30c primer sequence were designed and synthesized by the Shanghai Sangon Biological Engineering Co., Ltd. (Shanghai, China) (Table I).

\section{Method}

RT-qPCR assay. The collected PCa cancer tissue and PCa adjacent tissues was performed with total RNA extraction by using
TRIzol reagent, in strict accordance with the instructions for extraction. The concentration and purity of extracted RNA were detected by UV spectrophotometer (Hitachi, Tokyo, Japan). The OD value of the total RNA solution: A260/A280 ranged from 1.8-2.1, and if not, it was extracted again. The integrity of RNA was detected by $1 \%$ denaturing agarose gel electrophoresis. The total RNA was subjected to reaction system disposition and reverse transcription to cDNA by miRNA reverse transcription kit instructions (and stored at $-20^{\circ} \mathrm{C}$ for later use). ABI StepOne Plus fluorescence quantitative PCR instrument was adopted, and the reaction system was according to the instructions. Reaction conditions: $95^{\circ} \mathrm{C}$ for $5 \mathrm{~min} ; 95^{\circ} \mathrm{C}$ for $45 \mathrm{sec} ; 60^{\circ} \mathrm{C}$ for $60 \mathrm{sec} ; 72^{\circ} \mathrm{C}$ for $45 \mathrm{sec}$ and 45 cycles. U6 was used as internal reference. The experiment was repeated 3 times, and the results were analyzed by using the $2^{-\Delta \Delta \mathrm{Cq}}$ method (10).

Statistical analysis. The study used SPSS 20.0 software package (Version X; IBM, Armonk, NY, USA) to perform statistical analysis for all the results collected. GraphPad Prism 5 software was adopted for image rendering. The enumeration data are expressed as a percentage (\%). The Student's t-test was used for comparisons between the two groups. The measurement data are expressed as mean \pm standard error (SE), and the ROC curve of miR-29b and miR-30c was plotted. $\mathrm{P}<0.05$ was considered to indicate a statistically significant difference.

\section{Results}

Expression of miR-29b and miR-30c in PCa tissues. By RT-qPCR, we detected the expression of miR-29b in cancer tissues of PCa and adjacent tissues. The results showed that miR-29b was significantly lower in the PCa tissues $(1.57 \pm 0.48)$ than that in the paracancerous tissues $(2.94 \pm 1.08)(\mathrm{p}<0.05)$. However, the expression of miR-30c was also lower in $\mathrm{PCa}$ patients $(2.84 \pm 0.57)$ than that in paracancerous tissues $(5.24 \pm 0.42)(\mathrm{p}<0.05)$ (Figs. 1 and 2$)$.

The relationship between the low expression of $m i R-29 b$ and miR-30c and clinicopathological features in PCa patients. By comparing the expression of miR-29b and miR-30c and clinical data in PCa patients with cancer, we found that there was no correlation between age and smoking and TNM staging of miR-29b and miR-30c ( $>>0.05)$, while lymph node metastasis, bone metastasis, Gleason score were correlated with miR-29b and miR-30c expression $(\mathrm{p}<0.01)$ (Table II).

The clinical value of $m i R-29 b$ and $m i R-30 c$ in the diagnosis of $P C a$. To compare the expression levels of miR-29b and miR-30c in $\mathrm{PCa}$ patients with cancer tissues and adjacent tissues, we plotted the ROC curve, which indicated that miR-29b, AUC 
Table II. Correlation of miR-29b and miR-30c low expression and clinicopathological features of PCa patients.

\begin{tabular}{|c|c|c|c|c|c|c|c|}
\hline Clinical features & $\mathrm{n}$ & $\begin{array}{l}\text { miR-29b } \\
\text { expression }\end{array}$ & Statistics & P-value & $\begin{array}{l}\text { miR-30c } \\
\text { expression }\end{array}$ & Statistics & $\mathrm{P}$-value \\
\hline \multicolumn{8}{|l|}{ Age (years) } \\
\hline$\geq 55$ & 88 & $1.52 \pm 0.32$ & \multirow{2}{*}{1.389} & \multirow{2}{*}{0.166} & $2.74 \pm 0.42$ & \multirow{2}{*}{1.314} & \multirow{2}{*}{0.191} \\
\hline$<55$ & 99 & $1.59 \pm 0.38$ & & & $2.82 \pm 0.43$ & & \\
\hline \multicolumn{8}{|l|}{ Smoking } \\
\hline Yes & 180 & $1.42 \pm 0.59$ & \multirow[t]{2}{*}{0.133} & \multirow[t]{2}{*}{0.894} & $2.54 \pm 0.38$ & \multirow[t]{2}{*}{0.948} & \multirow[t]{2}{*}{0.344} \\
\hline No & 7 & $1.45 \pm 0.36$ & & & $2.68 \pm 0.47$ & & \\
\hline \multicolumn{8}{|c|}{ Lymph node metastasis } \\
\hline Yes & 104 & $1.53 \pm 0.35$ & \multirow[t]{2}{*}{2.092} & \multirow[t]{2}{*}{0.004} & $2.64 \pm 0.32$ & \multirow[t]{2}{*}{2.900} & \multirow[t]{2}{*}{0.004} \\
\hline No & 83 & $1.66 \pm 0.50$ & & & $2.88 \pm 0.34$ & & \\
\hline \multicolumn{8}{|l|}{ Bone metastasis } \\
\hline Yes & 133 & $1.43 \pm 0.27$ & \multirow[t]{2}{*}{3.955} & \multirow[t]{2}{*}{0.001} & $2.72 \pm 0.22$ & \multirow[t]{2}{*}{2.943} & \multirow[t]{2}{*}{0.004} \\
\hline No & 54 & $1.61 \pm 0.31$ & & & $2.84 \pm 0.32$ & & \\
\hline \multicolumn{8}{|l|}{ TNM staging } \\
\hline I & 55 & $1.72 \pm 0.31$ & \multirow{3}{*}{0.626} & \multirow{3}{*}{0.535} & $2.94 \pm 0.37$ & \multirow{3}{*}{2.895} & \multirow{3}{*}{0.058} \\
\hline II & 60 & $1.69 \pm 0.37$ & & & $2.88 \pm 0.22$ & & \\
\hline III+IV & 72 & $1.66 \pm 0.22$ & & & $2.82 \pm 0.24$ & & \\
\hline \multicolumn{8}{|l|}{ Gleason score } \\
\hline$\geq 8$ & 107 & $1.33 \pm 0.20$ & \multirow[t]{2}{*}{3.642} & \multirow[t]{2}{*}{0.004} & $2.44 \pm 0.29$ & \multirow[t]{2}{*}{3.315} & \multirow[t]{2}{*}{0.001} \\
\hline$<8$ & 80 & $1.44 \pm 0.21$ & & & $2.60 \pm 0.37$ & & \\
\hline
\end{tabular}

$\mathrm{PCa}$, prostate cancer.

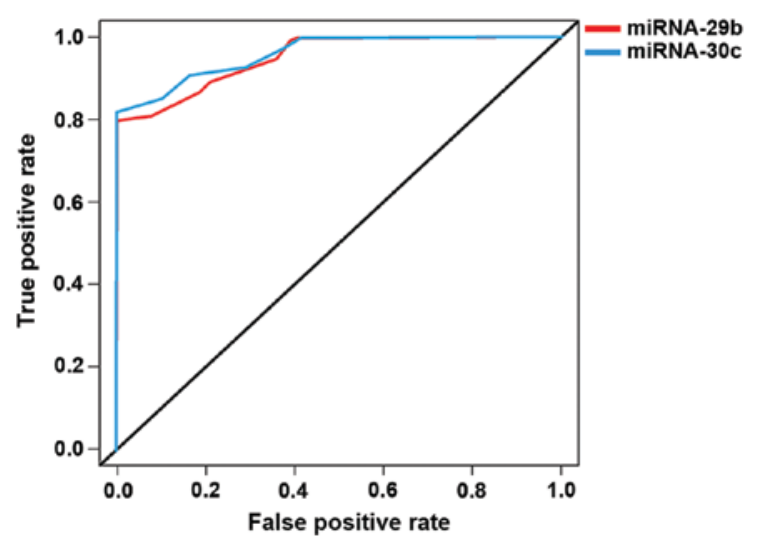

Figure 3. The clinical value of miRNA-29b and miRNA-30c in the diagnosis of PCa miRNA-29b AUC, 0.924; 95\% CI, 0.824-0.967, and miRNA-30c AUC, $0.944 ; 95 \%$ CI, 0.798-0.972. PCa, prostate cancer.

was 0.924 , and the $95 \%$ CI was $0.824-0.967$; the specificity was $84.4 \%$, and the sensitivity was $76.5 \%$; while for miR-30c, AUC was 0.944 , and $95 \%$ CI was $0.798-0.972$; the specificity was $80.2 \%$, and the sensitivity was $72.3 \%$. The two miRNAs were effective in differentiating cancer tissues from paracancerous tissues with high specificity and sensitivity (Fig. 3).

\section{Discussion}

As a malignant tumor that seriously affects the quality of life of men, the pathogenesis and causes of PCa are complicated (11).
Studies have found that the conditions of family heredity and environmental factors of $\mathrm{PCa}$ patients can lead to the occurrence and development of PCa, and the incidence in men under 50 is not high, but the incidence of PCa after the age of 50 years increases exponentially (12). Another study shows that (13) if the early PCa is timely treated, the prognosis will be better. PSA is widely used as a primary screening tool for $\mathrm{PCa}$ in early clinical diagnosis. However, PSA specificity is low; PSA increases in a variety of common prostatic diseases, and there is also considerable controversy regarding whether PSA testing can reduce PCa mortality. Therefore, we must find a more suitable biomarker to improve the diagnosis of $\mathrm{PCa}$, providing a more reliable method for clinical treatment and diagnosis.

miRNAs are a class of endogenous, non-coding, short-chain RNAs that degrade target genes and suppress translation of target genes, thereby silencing a gene transcription (14). Studies have shown that nearly $30 \%$ of the encoded proteins in the human body are influenced and regulated by miRNAs (15). miRNAs are differentially expressed in tumors, and can be repressed or promoted by the regulation of target genes. It has been reported in the literature (16-18) that the biological functions of miR-29b and miR-30c have an important effect on the pathophysiological and pathological changes of tumor cells, and play a regulatory role in various functions such as proliferation, differentiation and apoptosis of cancer cells.

In this study, we detected the expression levels of miR-29b and miR-30c in 187 cases of PCa by RT-qPCR, and found that 
the expression of miR-29b in cancer tissues was significantly lower than that in adjacent non-cancerous tissues. Similarly, miR-30c expression in cancer tissues was significantly lower than that in paracancerous tissues. Through the correlation analysis of the two miRNAs low expression and clinical records data, we found that lymph node metastasis, bone metastasis, and Gleason score were correlated with the expression of miR-29b and miR-30c, and by plotting ROC cure of miR-29b and miR-30c resulted in miR-29b AUC, 0.924; 95\% CI, 0.824-0.967, and miR-30c AUC, 0.944; and 95\% CI, 0.798-0.972. This is a good illustration that both miRNAs have a very good diagnostic value in the diagnosis of PCa. Forty-four patients with PCa were detected by RT-qPCR by Huang et al (19), they found that the cancerous tissues of $44 \mathrm{PCa}$ patients were significantly downregulated compared with the results of our present experiment, there were some differences but not significant, which may be caused by the difference of the patient's area and the experimental design, and the ROC curve plotted is basically the same as the ROC curve drawn by us. In the study by Zhang et al (20), miR-30c was found to be highly effective in inhibiting proliferation, migration and invasion of PCa cells. In the study of Steele et al (21), miR-29b was found to have low expression in PCa cells, and was significantly lower in PCa tissues than that in adjacent non-cancerous tissues. It was also found that $\mathrm{N}$-cadherin, Twist and Snail protein expression was significantly downregulated, which is also a good illustration that miR-29b and miR-30c are potential biomarkers for the treatment and diagnosis of $\mathrm{PCa}$.

However, this study also has some shortcomings. Only the expression of miRNAs in PCa tissues was examined, and did not follow up the patients. In addition, the sample size was small. Whether the regional differences would bias the experimental results is not known. Therefore, we hope that in future trials, we will increase the number of specimens, increase the control group of cancer adjacent tissue group and follow up the patients promptly in the hope of better verifying the correctness of this investigation.

In summary, miR-29b and miR-30c play an important role in the occurrence and development of $\mathrm{PCa}$, which is expected to become a novel marker for early diagnosis and prognosis of $\mathrm{PCa}$.

\section{Acknowledgements}

Not applicable.

\section{Funding}

No funding was received.

\section{Availability of data and materials}

The datasets used and/or analyzed during the present study are available from the corresponding author on reasonable request.

\section{Authors' contributions}

CZ drafted this manuscript. CZ and WW were responsible for the conception and design of the study. XH collected the patients' data, and revised the manuscript critically for important intellectual content. JZ and CJ analyzed and interpreted the data. All authors read and approved the final manuscript.

\section{Ethics approval and consent to participate}

This study was approved by the Ethics Committee of Jining First People's Hospital (Jining, China). Signed written informed consents were obtained from the patients or guardians.

\section{Patient consent for publication}

Not applicable.

\section{Competing interests}

The authors declare that they have no competing interests.

\section{References}

1. Wolff JM and Mason M: Drivers for change in the management of prostate cancer - guidelines and new treatment techniques. BJU Int 109 (Suppl 6): 33-41, 2012.

2. Sfanos KS, Yegnasubramanian S, Nelson WG and De Marzo AM: The inflammatory microenvironment and microbiome in prostate cancer development. Nat Rev Urol 15: 11-24, 2018.

3. Kenfield SA, Tat D and Chan JM: The potential benefits of diet and physical activity among active surveillance patients with low-burden prostate cancer. In: Active Surveillance for Localized Prostate Cancer. Current clinical urology. Klotz L (ed). Humana Press, Totowa, NJ, 2012.

4. Baade PD, Youlden DR, Cramb SM, Dunn J and Gardiner RA: Epidemiology of prostate cancer in the Asia-Pacific region. Prostate Int 1: 47-58, 2013.

5. Esfahani M, Ataei N and Panjehpour M: Biomarkers for evaluation of prostate cancer prognosis. Asian Pac J Cancer Prev 16: 2601-2611, 2015.

6. Marantz PR, Hall CB and Derby CA: Radical prostatectomy versus watchful waiting. N Engl J Med 353: 1298-1300, 2005.

7. Carthew RW: Gene regulation by microRNAs. Curr Opin Genet Dev 16: 203-208, 2006.

8. Iorio MV and Croce CM: MicroRNA dysregulation in cancer: Diagnostics, monitoring and therapeutics. A comprehensive review. EMBO Mol Med 4: 143-159, 2012.

9. Ferretti S, Patriarca S, Carbone A and Zanetti R: TNM classification of malignant tumours, VII edition 2009. Changes and practical effects on cancer epidemiology. Epidemiol Prev 34: 125-128, 2010 (In Italian).

10. Livak KJ and Schmittgen TD: Analysis of relative gene expression data using real-time quantitative PCR and the 2(-Delta Delta C(T)) method. Methods 25: 402-408, 2001.

11. White A, Joseph D, Rim SH, Johnson CJ, Coleman MP and Allemani C: Colon cancer survival in the United States by race and stage (2001-2009): Findings from the CONCORD-2 study. Cancer 123 (Suppl 24): 5014-5036, 2017.

12. Thorstenson A, Garmo H, Adolfsson J and Bratt O: Cancer specific mortality in men diagnosed with prostate cancer before age 50 years: A nationwide population based study. J Urol 197: 61-66, 2017.

13. Pokharel SS, Patel NU, Garg K, La Rosa FG, Arangua P, Jones C and Crawford ED: Multi-parametric MRI findings of transitional zone prostate cancers: Correlation with 3-dimensional transperineal mapping biopsy. Abdom Imaging 40: 143-150, 2015.

14. Ha M and Kim VN: Regulation of microRNA biogenesis. Nat Rev Mol Cell Biol 15: 509-524, 2014.

15. Carthew RW: Gene regulation by microRNAs. Curr Opin Genet Dev 16: 203-208, 2006.

16. Nishikawa R, Goto Y, Kojima S, Enokida H, Chiyomaru T, Kinoshita T, Sakamoto S, Fuse M, Nakagawa M, Naya Y, et al: Tumor-suppressive microRNA-29s inhibit cancer cell migration and invasion via targeting LAMC1 in prostate cancer. Int J Oncol 45: 401-410, 2014. 
17. Moltzahn F, Olshen AB, Baehner L, Peek A, Fong L, Stöppler H, Simko J, Hilton JF, Carroll P and Blelloch R: Microfluidic-based multiplex qRT-PCR identifies diagnostic and prognostic microRNA signatures in the sera of prostate cancer patients. Cancer Res 71: 550-560, 2011.

18. Ling XH, Han ZD, Xia D, He HC, Jiang FN, Lin ZY, Fu X, Deng YH, Dai QS, Cai C, et al: MicroRNA-30c serves as an independent biochemical recurrence predictor and potential tumor suppressor for prostate cancer. Mol Biol Rep 41: 27792788,2014

19. Huang Z, Zhang L, Yi X and Yu X: Diagnostic and prognostic values of tissue hsa-miR-30c and hsa-miR-203 in prostate carcinoma. Tumour Biol 37: 4359-4365, 2016.
20. Zhang J, Wang X, Wang Y, Peng R, Lin Z, Wang Y, Hu B, Wang J and Shi G: Low expression of microRNA-30c promotes prostate cancer cells invasion involved in downregulation of KRAS protein. Oncol Lett 14: 363-368, 2017.

21. Steele R, Mott JL and Ray RB: MBP-1 upregulates miR-29b that represses Mcl-1, collagens, and matrix-metalloproteinase-2 in prostate cancer cells. Genes Cancer 1: 381-387, 2010.

This work is licensed under a Creative Commons Attribution-NonCommercial-NoDerivatives 4.0 International (CC BY-NC-ND 4.0) License. 